\title{
Plasticity of intermediate mechanics students' coordinate system choice
}

\author{
Eleanor C. Sayre \\ Department of Physics, Ohio State University, Columbus, Ohio 43201, USA \\ Michael C. Wittmann \\ Department of Physics and Astronomy, College of Education and Human Development and Center for Science and Mathematics \\ Education Research, University of Maine, Orono, Maine 04469-5709, USA
}

(Received 15 March 2007; published 12 November 2008)

\begin{abstract}
We investigate the interplay between mathematics and physics resources in intermediate mechanics students. In the mechanics course, the selection and application of coordinate systems is a consistent thread. At the University of Maine, students often start the course with a strong preference to use Cartesian coordinates, in accordance with their prior physics and mathematics classes. In small-group interviews and in homework help sessions, we ask students to define a coordinate system and set up the equations of motion for a simple pendulum for which polar coordinates are more appropriate. We analyze video data from several encounters using a combination of Process/Object theory and Resource Theory. We find that students sometimes persist in using an inappropriate Cartesian system. Furthermore, students often derive (rather than recall) the details of the polar coordinate system, indicating that their knowledge is far from solid. To describe our work more precisely, we define a scale of plasticity and several heuristics for defining resources and their plasticity.
\end{abstract}

DOI: 10.1103/PhysRevSTPER.4.020105

PACS number(s): 01.30.1b, 01.40.Fk, 01.40.Ha

\section{INTRODUCTION}

As part of ongoing research into cognitive processes and student thought, we investigate the interplay between mathematics and physics resources in intermediate mechanics students. We collect data from student interactions to build models of student cognition, which are then used to inform curriculum development.

In this paper, we consider the question of how new ideas develop in students. We are particularly interested in the development of mathematical ideas within physics-in this case, the use of coordinate systems to describe a physical situation. In Sec. II, we review our theoretical framework, review how others within this tradition have dealt with the construction of new ideas, and introduce heuristics for recognizing resources and their plasticity. In Sec. III, we describe our research setting. Section IV forms the bulk of the paper, with an extended description of two students defining the coordinate systems used to describe a simple pendulum. The paper concludes with a brief discussion.

\section{THEORETICAL DEVELOPMENT}

We use theoretical perspectives from both mathematics education research (Process/Object ${ }^{1}$ ) and physics education research (Resource Theory $\left.{ }^{2}\right) .{ }^{3}$ In this section, we present an overview of Resource Theory and its connections to Process/ Object as extended by the recognize/build-with/construct (RBC) model for abstraction. ${ }^{4-7}$ We introduce the idea of plasticity, a continuum which extends Resource Theory to describe the development of resources. We then present heuristics for identifying resources and their plasticity in situ.

\section{A. Resource Theory}

Resource Theory is a constructivist schema theory which bridges neurocognitive models of the brain and results from education research ${ }^{8,9}$ to describe the phenomenology of problem solving. ${ }^{10}$ Resources are small reusable pieces of thought that make up concepts and arguments. In contrast to Process/Object's historical focus on conceptions of mathematical entities, research using Resource Theory has traditionally focused on the connections between different ideas in physics.

Because of Resource Theory's genesis in the knowledgein-pieces tradition, most examples of resources in the literature primarily focus on primitives. ${ }^{10}$ Some examples include "effect dies away," which describes the motion of a box sliding on a floor, the ringing of a struck bell, a person's motivation, and other phenomena. A mathematical equivalent exists in symbolic forms. ${ }^{11,12}$ Though most described resources are primitive and thought of as having no internal structure, in this paper, we describe a larger resource, coordinate systems, with much internal structure.

As originally published, ${ }^{2}$ resources were intentionally vaguely defined. Later papers elaborate on the theory and make more explicit connections between resources and other theories..$^{8,9,13-19}$ As Resource Theory has developed, different aspects of student cognition have been explored using Resources, including epistemology, ${ }^{13,16,20,21}$ metacognition, ${ }^{15}$ and the relationship between physics and mathematics content knowledge. ${ }^{17,22}$ Representations of linked resources have been described ${ }^{18}$ and made consistent with the model of coordination classes. ${ }^{23}$ These explorations have, in turn, enriched and made explicit some of the details of the original skeletal framework, rendering it more like a full-bodied theory.

As a theory, resources have been used in conjunction with epistemic games and frames, ${ }^{24}$ students' sense of physical mechanism, ${ }^{25}$ and student reasoning in nearly novel situations. ${ }^{16}$ It has been used to support curriculum development in areas of introductory algebra-based physics, ${ }^{26}$ intuitive quantum physics $^{27}$ and intermediate classical mechanics. ${ }^{28,29}$ Based on the literature and our own work, we 
summarize that individual resources and groups of resources have the properties listed below.

\section{Individual reusable thoughts}

Resources are small reusable pieces of thought that make up concepts and arguments. ${ }^{2}$ To be considered a resource, an idea must have sufficient duration and stability to be reused. Resources are individually nameable, such as coordinate systems or knowledge from authority. ${ }^{13}$ Note that in this paper, we will italicize the name of resources. Researchers name resources; students need not be aware of the resources used or their names.

Individual students hold resources; they are not socially negotiated (unlike social norms). ${ }^{30}$ Different people may each hold a version of a given resource, and the construction and details of use may differ among people.

\section{Two states}

Resources have two states: active and inactive. An active resource is being used; an inactive one is dormant. This twostate system harkens to neurology, in which neurons have two states. ${ }^{31}$

The physical context and cognitive state of the user determine which resources are available to be activated. The activation of resources occurs when their invocation, express or implicit, is used to support or form an argument.

Asking a child at the park "Where's the ball?" may activate resources for an activity (looking) as well as resources for balls as objects that are round and bouncy. The same question posed at bedtime may activate resources for the story time activity as well as princess or dancing resources. The choice of activated resources depends on the child's framing of the activity in which she is engaged. ${ }^{14,24,32}$

\section{Connection and activation}

In the ball question, multiple resources activate. These activations are not unconnected. Resources link with each other. These connections are commonly conceptualized as ball-and-stick style graphs. ${ }^{18}$ Each resource is a node in the graph, and each connection is a directional link. ${ }^{33}$ This network model is consistent with a model of coordination classes. $^{23}$

As an example, consider the motion of a tossed coin. ${ }^{18}$ Figure 1 shows a possible resource graph. In considering this question, you probably activated a velocity resource. Activating velocity may have activated actuating agency and then forces as you consider why the coin moves. The motion dies away as the coin nears the top and slows down. Of course, to think about the coin in this manner, you have already activated object (instead of money), and you are probably using part-for-whole to think about the center of mass motion while ignoring spinning or flipping.

Just as neuronal links may be excitatory or inhibitory, links between resources may promote or demote activation. ${ }^{8}$ If the network tends to have the same structure repeatedly, then it is "stable." If not, then it has been built "on the fly." Stable networks consist of resources that are cognitively "nearby" each other: they tend to activate together. ${ }^{33,34}$

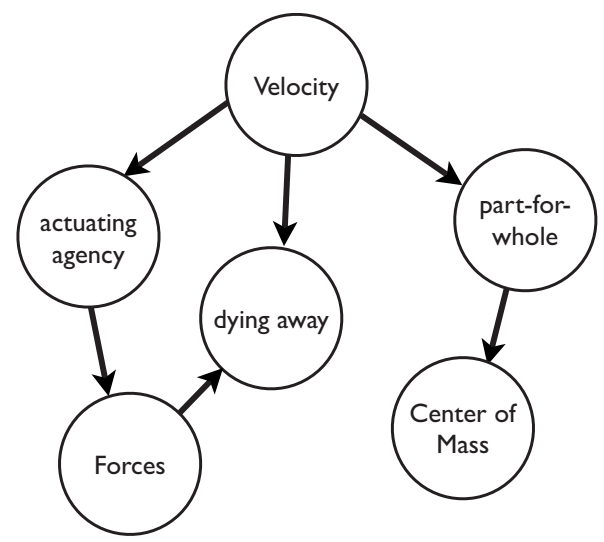

FIG. 1. Resource graph for the motion of a tossed coin. Only activated resources are shown.

\section{Internal structure}

In the ball question, one resource that could activate is princess. Princess is not a primitive idea; it has constituent ideas for what princesses are, their roles in fairytales, appropriate princess dress, etc. As a resource, princess illustrates that resources are nestable; ${ }^{33}$ they may have internal linked structure made up of other resources. In the resource graph for the tossed coin (Fig. 1) forces is certainly not primitive, but it links to other resources (like actuating agency) which are more so.

Many concepts described in the literature (such as force) have a complicated internal structure that is explorable but perhaps not explored by the user when used as a resource. In contrast, many primitives (such as diSessa's phenomenological primitives, ${ }^{10}$ known as "p-prims") may have internal structure that is no longer explorable. A large body of literature has identified both concepts ${ }^{35}$ and some kinds of primitives. ${ }^{10}$ Not all resources with internal structure are concepts. We describe both resources with and without internal structure in this paper.

\section{B. Development of resources}

Several problems exist with the summary given above. Foremost for the topic of this paper, there is no agreement on a developmental path for the creation of new resources. A primitive might develop early in childhood: actuating agency is learned early by infants, and useful throughout life, for example. Other, less primitive resources develop much later in life. Refined physics ideas, readily available to an expert and applicable in a variety of settings (be it tricks of integration, knowledge of subatomic particles, or ad hoc rules about lattice structure), are obviously not learned in childhood. Several suggestions have been made for how new resources come to be. We consider four here: cueing and reliability priorities of p-prims, development of symbolic forms as p-prims in mathematical systems, reorganization of resource graphs, and framing within a resource perspective.

\section{Cuing and reliability priorities of p-prims}

Typically, we use a simplistic description of p-prim activation to describe what happens when a user reasons, for 
example, about the physical actions of a moving object interacting with its surroundings. This process of activation is often simply thought of as a "recognition" that the particular primitive is relevant and useful at that moment. In diSessa's seminal work ${ }^{10}$ on p-prims, he describes two ways in which recognition plays a role, cuing priority and reliability priority. These help define how recognition occurs and depend on the details of a scenario being analyzed.

Cuing priority describes how likely a p-prim is to be activated in a given situation. A low cuing priority means that a p-prim is highly unlikely to be activated in a given setting. For example, the coin toss problem activates objectlike ideas about center of mass rather than moneylike ideas about value. In another example, setting determines if asking about a ball activates ideas about a princess or a park. Cuing priority is thus a context dependent measure that describes the likelihood of an original activation.

Reliability priority describes how likely a p-prim is to remain activated once activated in a given setting. If the reliability priority is high, then other connected p-prims are likely to be activated as well. If the reliability priority is low, then even strongly connected p-prims are unlikely to be activated in that setting because the p-prim which could have led to their activation quickly deactivates in that setting.

By considering ways in which cuing and reliability priorities change, one can describe how p-prims adapt over time to become associated with new settings (or other p-prims) more consistently (both in activation and length of activation). However, this model assumes that the p-prims themselves are not changing, even though some of their properties may be; the basic idea of a p-prim (such as "dying away") does not change even as it is associated with new settings and other primitives.

In this paper, we implicitly use cuing and reliability priorities as we expand on the ways in which the properties of resources indicate whether they are easily available for use in a given setting. We introduce several measures (described in Sec. V) to account for different types of change that might occur in resources. Many of these extensions depend on the fact that resources are not necessarily primitive and may have considerable substructure.

\section{Forms as primitives in a symbolic context}

In his Ph.D. dissertation, ${ }^{36}$ Sherin stated that new resources "develop, in part, out of existing resources" and "once the new resources have developed, connections may remain between the new resources and the previously existing resources" (p. 152). Specifically, he argued that one kind of resource, a symbolic form, develops through the connection of p-prims to the abstraction of symbolic experience. Much as p-prims such as actuating agency arise out of everyday experiences in the physical world, a primitive sense of base plus change arises from one's manipulation of numbers and symbols beginning early in one's use of mathematics. Importantly, Sherin ${ }^{11,36}$ described the reinforcement that occurs when certain forms are used frequently and the associated p-prims are activated. In such a situation, the resource becomes more and more powerful in one's reasoning. Sherin's description ${ }^{11,36}$ is consistent with diSessa's cuing priority ${ }^{10}$ but is applied to the development of new ideas, namely, symbolic forms.

We make two amendments to Sherin's work ${ }^{11,36}$ in this paper. First, we consider resources at a different level than Sherin ${ }^{11,36}$ did. Where Sherin ${ }^{11,36}$ talked about a rather largescale cognitive resource of intuitive physics or algebra physics, we speak of resources in a way more closely aligned with Hammer's description of "fine-grained" reasoning elements or Minstrell's view ${ }^{37}$ of "lightly abstracted" versions of what students say. Second, and most importantly, we give additional detail on the ways in which connections between resources and between resources and settings indicate a fluidity in understanding.

\section{Changes in mesoscopic resource graphs}

Because resources are nestable, resources might be made up of graphs containing other resources and these graphs may link to resources of different sizes. One form of development of a resource might be the reorganization of a resource graph into a new (and stable) graph. Such a description is consistent with changes in cuing priority. Also, one can think of resource graphs as containing only resources with a high reliability in a given setting (and are therefore reliably linked with other resources).

In a previous work, one author (Wittmann ${ }^{18}$ ) described linked graphs of resources and the ways in which changes to these graphs might be used to describe several forms of conceptual change. Two processes of conceptual change originally defined by Demastes ${ }^{38}$ were described in terms of resource graphs. In an incremental change, one resource in a graph changes while the others stay the same. Several consecutive but independent incremental changes are possible. In a cascade change, the series of incremental changes is not independent, but each depends on one (or more) of the previous changes. In another process, a large resource graph might split in two as ideas are differentiated. In a state where dual constructions are available to the user, different resource graphs are available at the same time, activated differently depending on a nuanced reading of the context.

We did not originally intend to use resource graphs to define the creation of new resources. Instead, the assumption was that the resources that form resource graphs are solidly understood and easily available to the user. We reconsider our original perspective in this paper.

\section{Framing and frames}

Another perspective exists when considering how sets of resources are applied in a given setting. For example, during a physics laboratory, a student may use one set of resources for manipulating the equipment and another for coordinating her groupmates. In the former frame, she uses resources for uncertainty calculations and repeatable measures, while in the latter frame she uses resources for allocation of labor and gossip management.

Hammer et al. ${ }^{14}$ and Tuminaro ${ }^{17}$ described such a process of recognizing which resources to use within a setting as "framing," building primarily on Tannen and Wallat, ${ }^{32,39}$ frames deal with "structures of expectation:" 32 the way par- 
ticipants in a situation figure out "what's going on here?" In a resources perspective, such a question is answered by defining which resources are activated in that setting. Framing, then, is the active bringing together of one or several resources in an effort to understand and work within a situation.

Framing may be able to show the development of resources, though this development is not detailed in the literature connecting framing to Resource Theory. ${ }^{14}$ Because framing is meant to bring together locally coherent sets of resources, a frame might function like a protoresource (in that a quickly built resource graph has the possibility of being reified into a single resource). Over time and with reinforcement, the protoresource may compile to produce an actual resource, ready to be activated as is. Consider, for example, a physics student learning to apply the wave equation. This student might have to deliberately choose to use separation of variables when solving a second-order differential equation because the method is not readily recruited into her wave behavior frame. Over time, using separable to describe equations becomes commonplace; the frame readily recruits it. In other words, framing a problem as "a wave problem" requires use of separable. This assumption of resource development as the development of regularly applicable frames depends heavily on the description of resources with internal structure and frames as reified acts of framing which come to be resources in and of themselves.

While recognizing that framing is a valuable way to address how students come to terms with unfamiliar situations and contexts, we have chosen a different type of analysis. Framing is typically described as an activity which recruits existing resources to address problems or specific contexts. We, instead, consider the development of the resources alone. We track resources over time, looking at how interresource and intraresource links develop in strength and availability. We believe that our description of resource development (as described in Sec. V) and framing are most likely complementary, but it is presently unclear how to connect them formally. ${ }^{40}$

\section{Plasticity}

The plasticity continuum ${ }^{41}$ is an extension to Resources which describes the generation and development of resources. The two directions in the continuum are more solid and more plastic. (We think of "more plastic" things as if they are a soft gel and not yet hardened.) A solid (explorable) resource can be considered a durable concept; its connections to other resources are plentiful, and its internal structure is unlikely to change under typical use. ${ }^{42}$ Plastic resources, in contrast, are less durable in time or less stable in structure; they are not the reified objects that more solid resources are. The more plastic a resource is, the less likely the user is able to apply it to new situations and the more explanation is needed to justify and explain its use. The more solid a resource is, the more likely the user is to refer to the resource in diverse contexts without explaining its internal structure. As with all resources, the plasticity of a resource is independent of its veracity.
We use the RBC model ${ }^{4-7}$ to inform and improve the plasticity continuum, noting that the RBC model was originally intended to describe the reification and abstraction of concepts and not all resources need be thought of as concepts. The RBC model proposes three epistemic actions through which abstraction occurs and which may be inferred from observable behavior. These three actions-recognizing, building-with, and constructing - are dynamically nested.

Recognizing, the simplest action of the three, occurs when a student realizes that a "familiar mathematical notion, process, or idea is inherent in a given mathematical situation."4 These recognized cognitive objects are akin to resources (or other similar constructs). Recognition is thus synonymous with activation of resources. The specifics of which resources are recognized give insight into students' thought structure and may depend on a student's framing of a situation. Ease of recognition is therefore a marker of solidity.

Once a familiar idea has been recognized, a student may build-with that idea to solve a local goal, such as solving a problem or justifying a statement. Several resources may need to be recognized and built-with at once. Under Resource Theory, activated resources form a web or graph that may be built on the fly. Such resources require relatively high reliability priority in order to exist long enough to allow for graph building. Because building-with and recognizing are two separate actions, the RBC model allows us to describe behavior when students mention an idea but do not appear to know what to do with it. In diSessa's formulation, ${ }^{10}$ a resource with high cuing priority and high reliability priority might simply not be strongly connected to many other resources in that given context. Such a resource is recognized but not yet available for a building-with action.

In contrast to building with, constructing has purpose and duration beyond solving a local goal. Constructing creates a less-local, more abstract entity. As a construction becomes more durable, it becomes more consolidated and is no longer necessarily built on the fly. The new-formed resource may be quite plastic, but as further constructions are added to it and as it compiles further, it can become more solid. It becomes a resource in its own right and therefore can be recognized or built-with in later local goals. Thus, constructing is a mechanism for increasing the solidity of specific resources. This language is similar to that of framing. However, it includes an element of abstraction across contexts which is distinct from a context-specific frame-based approach. This abstraction may lead to a more general and reliably activated frame in a given context. Extremely solid resources-rigid resources-have been so tightly compiled that their internal structure is not readily accessible to the user.

The plasticity continuum thus speaks to resources' stability of structure, their durability in time, and their connectedness to other resources. In many cases, these dimensions tend to follow each other, and plasticity can be a useful indicator. Should these properties diverge, they must be specified individually. In cases like that, plasticity is too coarse a tool for analysis. In Secs. III-V, we introduce heuristics for identifying resources and their plasticity, then apply them to a case from intermediate mechanics further detail the theory. 


\section{Heuristics}

Having presented both a summary of Resource Theory and several ideas for how new resources might develop over time, we now suggest a collection of heuristics for identifying resources in situ. Our heuristics differ from those presented by $\operatorname{diSessa}^{10}$ for identifying p-prims because resources and p-prims bear enough differences that a different list of heuristics is desirable. A short list of heuristics for resources and their plasticity follows. These formalize and summarize points made previously in the paper.

The following heuristics describe how one can find "classical" resources: resources that are generally solid.

R1. Resources are reusable. For an idea to be considered a resource, it must have sufficient duration to be reused. Thus one appearance of an idea is suggestive, but insufficient, to term that idea a resource. ${ }^{43}$

R2. Resources may be referred to without exploring internal structure. It may be that the internal structure is unavailable, such as the case of primitives, or it may be that it is simply not currently explored by the user, as in the case of some concepts. In either case, the referring need not be explicit: resources may activate without being explicitly called.

R3. Resources are nameable by researchers. A resource is a discrete bit of thought. While it can be alluring at times to refer to "resources relating to a coin toss," for example, unspecific language of this nature does not identify the specific resources in question. This amount of detail is not always desirable, but it is possible in principle. Note that users need not name-or even be explicitly aware of - all the resources that they use.

R4. Resources activate. If a resource is not apparent in a given situation, that failure is not necessarily indicative that the resource does not exist, merely that it did not activate. One (or more) of the following conditions may be preventing its activation: another resource may be blocking it; the context may not be linked to it; or it may not exist. To prove a lack is difficult. Only over many situations, in varied contexts, is it safe to say that a resource which has never activated does not exist. ${ }^{44}$

More plastic resources present more difficulty, especially in items R1 and R2. Using the RBC model, we can extend R1-R4 to find evidence of resources' plasticity. We propose the following heuristics for defining the plasticity of resources. Examples for many are given elsewhere ${ }^{41}$ and in this paper:

P1. Ease of use. The more solid a resource is, the more easily it can be recognized or built-with. This ease of use is directly related to the number and strength of connections that a resource has. Well-connected resources are more likely to activate in a variety of situations.

P2. Recency of construction. Often, but not always, the more recently a resource was constructed, the more plastic it will be. Counter examples include infrequently used resources, which may be old but plastic (like a physics professor's criterion for critically damped harmonic motion), or recently constructed "flashbulb" resources, which are so vivid that, despite their newness, they are etched solidly upon the mind. (Flashbulb resources are like flashbulb memories. ${ }^{45}$ )
P3. Elaboration needed to evaluate. Users need to explicitly test plastic resources against other (often more solid) resources to determine if the plastic ones should be used in a given context. These tests often take the form of elaborative sense making. In contrast, solid resources can be apprehended whole and are often quickly recognized without elaboration.

P4. Justification. Because plastic resources often are tested against solid ones, solid resources can justify the use of plastic ones. The degree to which a resource justifies another can be used to see how nearby ${ }^{16}$ the two resources are and to see their relative solidity.

P5. Rejustification or rederivation needed for extended use. In a long episode, as resources fall out of working memory, very plastic resources may need to be rebuilt or rejustified. In contrast, more solid resources can be rerecognized quickly.

These heuristics extend the ideas described in Sec. II B. They describe elements of observables within the measure of plasticity while also combining these variables into a single measure. In Secs. III and V, we present data from an intermediate mechanics class to support a plasticity-based analysis of student reasoning.

\section{RESEARCH SETTING}

Intermediate mechanics is a particularly rich place to study the interplay between physics and mathematics ideas, as students often enter with a solid intuitive grasp of the physics (which may be incorrect), but have not yet applied sophisticated mathematics. At the University of Maine, intermediate mechanics is a one-semester physics course which meets for three $1 \mathrm{~h}$ periods each week. Generally, one of those periods is devoted to small-group work on researchbased guided-inquiry tutorials. ${ }^{46}$ The other two are lecture based. In the work described in this paper, the course generally followed a textbook ${ }^{47}$ with a typical intermediate mechanics schedule, starting with air resistance and continuing to damped and driven harmonic motion, energy considerations, Lagrangians, and rotational motion. Typically, about half of the students are concurrently enrolled in differential equations; the other half have already taken it.

One thread that runs through the entire course is the selection and application of appropriate coordinate systems. Upon starting the course, many students have a strong preference to use a Cartesian coordinate system where the positive directions are up and to the right. However, many problems in physics can be made simpler through using other coordinate systems. For example, finding the position of a pendulum as a function of time is simplest using polar coordinates. As these students develop as physicists, choosing appropriate coordinates for a problem becomes more important.

\section{A. Problem involving coordinate systems}

To investigate students' developing understanding of coordinate systems, as well as other questions, we collect video data from a variety of sources, including informal group help 


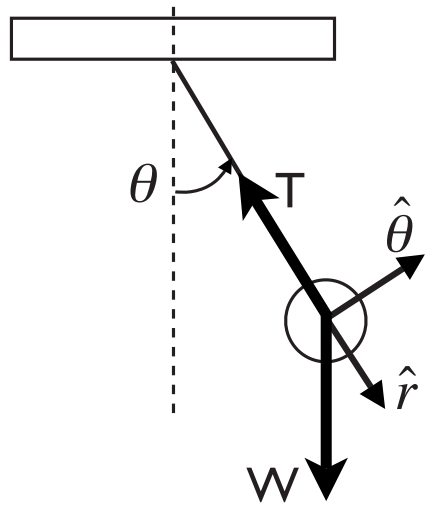

FIG. 2. The forces on a simple pendulum, with a physicist's polar coordinate system shown.

sessions, weekly small-group short interviews, and class discussion. We also collect written data in the form of ungraded (postlecture, pretutorial) pretests, homework assignments, and exams. In this paper, we focus on video data from one pair of students during short group interviews in weeks 4 and 10 during the Spring 2006 semester. As part of a larger study, in which a majority of the class was interviewed in small groups on a weekly basis, we pick out this pair because of the amount of time they spent discussing the choice of coordinate systems for the simple pendulum.

The two students, "Derek" and "Wes," volunteered to be interviewed together. Derek was a conscientious student who submitted thorough solutions to assigned problems. He started the semester averse to small-group tutorial work and finished a loyal supporter. In contrast, Wes rarely submitted complete solutions and had a poor work ethic. At times, he appears to enjoy being ornery. They were good friends and enjoyed mutually abusive banter. The playful tone of their interactions is not always evident in transcript.

In both interviews, students are presented with the same problem: given a (drawing of a) simple pendulum (Fig. 2, with polar coordinates shown in a way students did not see), find the position of the pendulum bob as a function of time. So that students do not spend time figuring out the forces on the bob, and to predispose the students into thinking of forcebased solutions, the forces on the bob (a weight force and a tension force) are given diagrammatically. So as not to predispose students into choosing a particular coordinate system, the forces are not described as being "vertically downward" (weight force) or "radially inward" (tension force).

To solve for the position of the bob as a function of time, a physicist might first write Newton's second law for the system, a vector second-order differential equation. The physicist would then choose a polar coordinate system as shown in Fig. 2. This coordinate system takes advantage of the natural geometry and symmetry of the situation, and it is a calculationally easy choice. With the coordinate system in place, the vector equation of motion can be split into two scalar differential equations and then solved. As the focus of the interviews was on the coordinate system choice, the students were not expected to solve the differential equations.

We note that students in the intermediate mechanics class have encountered the pendulum problem in some detail in a calculus-based introductory physics course. The pendulum is but one (relatively simple) example of harmonic motion, one of the most important models taught in an undergraduate physics major. We expected students to be familiar with the problem but to have forgotten the specifics of the modeling they previously did.

\section{B. Resource structure of coordinate systems}

Graphs can be used to represent multiple resources activated together. ${ }^{18}$ Just as resources can be grouped into graphs, an individual resource can also be made up of subgraphs containing other resources. The scale of such nested resources is often not clear. As researchers, therefore, we choose a level of specificity to examine, noting that other levels are possible and may yield interesting results. We designed the research task to match our investigation of the structure and development of resources.

We break the coordinate system resource into three subgraphs of resources nested within it: property resources, which describe general properties that coordinate systems bear; use resources, which describe when to use coordinate systems and which coordinate systems to use; and case resources, which hold the specifics of given coordinate systems. An example of resources in each subgraph is available in Table I. The exact breakdown of which resources belong in which subgraphs, as well as the intersubgraph and intrasubgraph connection details, are user specific and time specific. However, naming one possible set of components and their interplay gives a baseline against which users' ideas can be tested.

Using our breakdown of subgraphs within coordinate systems, it is possible to examine which resources activate in given situations and show intra-coordinate systems linkages. It is unreasonable to expect that all of these resources would activate in every episode; typically, only a few need be active, depending on the context.

To explore these resources, consider a ball rolling down a hill. One possible coordinate system is aligned parallel to the hill, positive downward and zero at the top of the hill. In choosing this coordinate system, you activated directionality to set the positive direction and value to set the zero at the top of the hill. You also-likely implicitly_chose to use one coordinate via numberline because only one coordinate is needed to span the ball's likely motion.

Of course, this coordinate system is explicit, but it is not inherent in the motion of the ball: we chose it. Another possible coordinate system is to have the positive direction be vertically upward, with zero at the bottom of the hill. The choice to use one or the other of these systems is somewhat arbitrary considering that the same information can be gleaned from either; they are equivalent. However, depending on the question at hand, it may be more calculationally easy to prefer the vertical coordinate (if we were using energy conservation, for example) or the parallel one (if we were finding the time to reach bottom, for example). Calculational ease, like so many other resources, might contain a complicated internal structure. However, we note that it can be treated as a resource both in the Minstrell ${ }^{37}$ sense of 
TABLE I. Subgraphs in coordinate systems. Each resource is described based on its application within coordinate systems. Noncoordinate system applications have more general descriptions.

\begin{tabular}{|c|c|c|}
\hline Properties & Directionality & Positive and negative, forward and backward \\
\hline & Orthogonality & $\begin{array}{l}\text { Each coordinate cannot be obtained through linear } \\
\text { combination of other coordinates in the same } \\
\text { system. Related to independent. }\end{array}$ \\
\hline & Span & $\begin{array}{l}\text { A set of all coordinates expresses all possible } \\
\text { dimensions of the space. }\end{array}$ \\
\hline & Equivalency & Different coordinate systems are interchangeable \\
\hline & value & quantities that can be measured and labeled. \\
\hline \multirow[t]{6}{*}{ Use } & Choice & Coordinate systems must be chosen \\
\hline & Explicitness & Use may be implicit or explicit \\
\hline & Natural & "Preferred" coordinate system based on geometry \\
\hline & Ease & $\begin{array}{l}\text { Preferred coordinate system based on calculational } \\
\text { ease }\end{array}$ \\
\hline & Arbitrariness & Choice of coordinate system is not predetermined \\
\hline & Consistency & $\begin{array}{l}\text { Within a problem, coordinate systems should not } \\
\text { change }\end{array}$ \\
\hline \multirow[t]{7}{*}{ Case } & Cartesian & A rectilinear coordinate system $(x, y, z)$ \\
\hline & Polar & $\begin{array}{l}\text { A circular coordinate system in two dimensions }(r \text {, } \\
\theta)\end{array}$ \\
\hline & Spherical & $\begin{array}{l}\text { A circular coordinate system in three dimensions } \\
(r, \theta, \phi)\end{array}$ \\
\hline & Numberline & A one-dimensional coordinate system \\
\hline & Diagrammatic & $\begin{array}{l}\text { Used to visually answer the question "Where } \\
\text { is...?" }\end{array}$ \\
\hline & Spaces & Phase space, vector space, state space, etc. ${ }^{a}$ \\
\hline & Graphs & $\begin{array}{l}\text { Often Cartesian representation of one or more } \\
\text { nonpositional variables }\end{array}$ \\
\hline
\end{tabular}

aNote that while these coordinates are not necessarily positions, the language used to describe their systems is locationlike.

"lightly abstracted" versions of what students say (as shown below) and in the sense that one can activate ease instead of choosing to make things hard for oneself. We often see students failing to invoke ease in their problem solving. In this paper, we use ease as a shorthand to refer only to calculational ease, noting that calculational ease may be only a subset of all things easy.

In this paper, we focus on only a few examples of resources which are closely tied to the pendulum problem. The case resources commonly activated in this problem are Cartesian and polar. These are often activated because they are natural to the geometry or easy to use mathematically. Finally, the issue of span arises in determining whether the chosen coordinate system can actually describe the entire system (and the space in which movement occurs) appropriately. Other resources are also commonly activated in this problem, notably arbitrariness, equivalency, and orthogonality, but we do not discuss their contributions here.

\section{DATA}

Though coordinate systems contains a large number of other resources, not all of them are activated in the inter- views discussed here. In addition, a great many resources unrelated to coordinate systems are activated. To help focus the discussion, we restrict the analysis to looking at the activation of and connections between five resources within coordinate systems (polar, Cartesian, natural, ease, and span) and the plasticity of only two resources (polar and Cartesian) for each student. In the process, we draw resource graphs of these five resources for each student.

\section{A. Preliminary resource graphs and plasticity (week 4)}

In the first interview (week 4), the interviewer presents the problem. Wes and Derek immediately launch into a discussion of Newton's second law, its applicability, and energy considerations. After several minutes of discussion without reference to a coordinate system, the TA asks, "What coordinate system are you using?" (line 129). (These line numbers are consistent with the start of the interview and the full interview transcript is given in the online appendix.) When her question is met with silence, she continues, "Let's make one." 


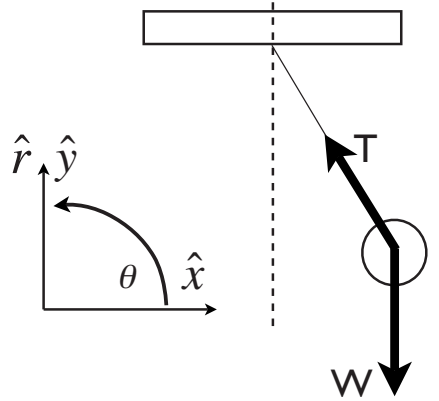

FIG. 3. Two coordinate systems from Wes. At first, he draws the $\boldsymbol{x}$ and $\boldsymbol{y}$ coordinates. In response to Derek's polar suggestion, he replaces $\boldsymbol{y}$ with $\boldsymbol{r}$, labels the angle between $\boldsymbol{r}$ and $\boldsymbol{x}$ as $\theta$, and erases $\boldsymbol{x}$.

\section{Polar, Cartesian, and ease (week 4)}

We first show the level of plasticity in polar resources of Wes and Derek. After the TA question, the following conversation occurred:

Wes: (Points) He loves this. We may not use it. (Draws Cartesian coordinates) Jeezum crow, that's an $x$.

Derek: Out of curiosity, why not use polar?

Wes: Eh?

Derek: Why not use polar? We're dealing 145

Wes: We're going to deal with $T$, we're going to deal with this, $L$.

Derek: Right, that's all constant, though.

In line 139, Wes draws a Cartesian coordinate system where positive is up and to the right. When he grumblingly redefines his system in response to Derek's suggestion of polar coordinates, ${ }^{48}$ he relabels the $y$ axis as the $r$ axis and defines the angle between the $x$ axis and $r$ axis as $\theta$ (Fig. 3). It is notable that some elements of Wes's polar system agree with mathematical convention: the two coordinates are $\boldsymbol{r}$ and $\boldsymbol{\theta}$, and $\boldsymbol{\theta}$ is measured counterclockwise from the right horizontal line. A conventional physical choice for this problem measures $\boldsymbol{\theta}$ counterclockwise from the downward vertical line, a quarter-circle phase difference. Both choices are valid. That Wes chooses mathematics convention, not physics convention, is not surprising given that his primary experience with polar coordinates has been in mathematics classes, not physics classes, even though he has most likely solved this problem in polar coordinates in his previous year's introductory physics class. It is also quite possible that he simply used the existing coordinates and redefined them but found no conflict between his redefinition into polar coordinates and the familiar mathematical language.

In this interaction, Wes chooses Cartesian because it is a natural choice. Derek argues that polar is more calculationally easy because only the angles change not the radii. Because only one coordinate is changing (a statement of span), the system can be reduced to a numberline-like problem. By heuristic R3, we observe that the two are activating different resources at this point. Each resource most likely has consid-

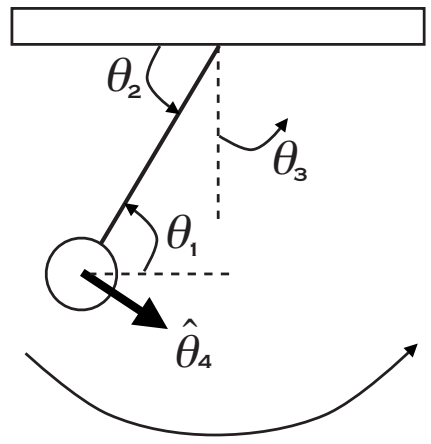

FIG. 4. Derek and Wes use four definitions of $\theta$ at different points in the two miniviews. Contrast their choices with the physicist choice presented in Fig. 2.

erable internal structure, which seems not to be relevant to either Wes or Derek. Instead, they justify their actions based on natural and ease, respectively, and move on.

It is notable that neither Wes nor Derek feel a need to explicitly define their zeros for either coordinate and consider the coordinate system discussion closed at this point: Wes immediately moves on to ask the TA if they are looking for the position as a function of time.

To return the discussion to coordinate systems, the TA asks Wes and Derek to apply their coordinate system, which is drawn next to the sketch of the pendulum (as in Fig. 3), to the sketch itself:

TA: $\quad$ Tell me more about this coordinate system you, you set up here.

185 Wes: Just polar.

TA: Kay....What direction is the-

Wes: Positive.

TA: In this picture? Yeah, which way is it.

Wes: This is positive theta.

Counterclockwise.

190 TA: Which way is this, $r$.

Wes: $\quad r$ is (gestures to the pendulum string on the diagram). If-what?

Derek: It really doesn't make a difference how you define it.

The speed of Wes's response, coupled with its brevity, indicates that he is using polar whole, without extensively deriving directions and zeros for the two coordinates. Wes is working at the level of choosing among prebuilt coordinate systems rather than constructing a coordinate system from scratch. Wes labels the polar angle as measuring counterclockwise from horizontal to the position of the bob $\left(\boldsymbol{\theta}_{1}\right.$ in Fig. 4). Just after this clip, both students continue, readily volunteering that $\boldsymbol{r}$ should be measured outward from the attachment point of the pendulum.

Derek's statement at line 193 is an expression of arbitrariness: it does not matter which direction is positive. Because Wes's definition of $\boldsymbol{\theta}$ indicates a curved path, but unit vectors are always drawn as straight lines, the TA presses Wes to show the direction of $\boldsymbol{\theta}$ at the instant shown, hoping that he will choose a direction tangent to the path. Wes demurs, asserting again that $\boldsymbol{\theta}$ is "counterclockwise."

We interpret this interchange about defining the system as showing Derek and Wes using different coordinate systems: 
polar (because it is easy) for Derek and Cartesian (because it is natural) for Wes. Furthermore, polar coordinates are connected to physical examples for Derek. They are less connected for Wes, for whom Cartesian coordinates are "traditional" (line 259) for any given problem and polar coordinates are not well defined. This indicates differing levels of plasticity: Cartesian coordinates are more solid for Wes, polar coordinates more plastic, and polar coordinates are more solid for Derek than they are for Wes. However, we do not yet have evidence to compare the plasticity of Derek's polar resource to Wes's Cartesian resource.

\section{Position, time, and span (week 4)}

After reciting positive directions for the TA, the students feel that their coordinate system definition is sufficient again. They quickly move on to solving the "real problem:" finding the position as a function of time. They discuss the graph of position vs time ("some sort of sinusoidal pattern" in line 230), whether mass is important, and whether they remember the formula for period.

The TA brings them back to discussing their coordinate system choices by asking them to draw their system on the board. Wes petulantly interjects with "Does it even matter?," an expression of arbitrary. He seems to object to the TA's continued harping on the matter of a coordinate system when he wants to move on to the "real problem."

Note that after the coordinate system was first mentioned and the pair decide on a polar system, Wes quickly moved to asking about the position as a function of time.

165 Wes: That's good. So you would want an equation that is any position in terms of time.

TA: Well...the position of the...yeah.

Wes: The position of this-

170 TA: In terms of time, yeah.

Wes: Now, do you want position in terms of this way (gestures horizontally on the diagram)? Or this...

Derek: Do you want like $x, y$ position?

175 TA: All, all I want to be able to do is tell you where it is-

Derek: It's much easier to use angles.

TA: - at any time.

Wes: Yeah.

180 Derek: Hence, why it's easier to use angles.

Wes: To just know where it is. Totally.

Wes's words and gestures indicate that he is still thinking about Cartesian as the most natural system for this problem. In contrast, Derek strongly holds that "angles"-a reference to polar systems - are easier.

Wes returns to his question of position as a function of time later in the miniview, starting with a petulant comment about arbitrariness. Wes is still using Cartesian to describe this system. In line 259, Wes says, "I would say if we had it in the traditional $x, y$, I just don't know where to go from...the start point."

Wes's activation of Cartesian as the most natural system for any problem — a "traditional" or default system-is getting in the way of solving for the position as a function of time. The TA asks him to consider how to solve the problem using polar coordinates. Wes responds that he would "just break it down into [redacted], um, arc lengths, kinematics equations with forces, and do some trig" (line 264). Derek eggs him to do so, and Wes grabs the marker, narrating his ideas. Some intervening lines have been omitted.

Wes: And work out all the math. Hey, I don't want to work out all the math. (redacted) (grabs marker). Well, let's say this is $L$ and say this is here, and this is a triangle, and that's a right angle, and that's some other distance I'm not going to name, yet, cause I don't feel like it. But...this is our theta up here. The sine theta...(writes). Yes, no?

280 height (pause) is whatever distance, $L$, minus this. That's our starting displacement from wherever it's going to be at the bottom. Then I'd use energy and figure out what it's speed would be at the bottom. But...we'll stay away

285 from energy, I guess. (Pause) 'Cause my mind's trying to be connected to...we want something to tell its position with respect to time. (Pause: 15:00) (continues).

During Wes's extended argument, Derek is largely silent and working privately on an unrelated problem. In analyzing Wes's argument, we break the argument into three segments, the first two of which are shown in the clip above. In the first part (lines 267-275), Wes labels lengths, heights, and angles in the problem. In the omitted lines above (lines 276-278), he seeks Derek's social agreement on his naming scheme. In the third part of Wes's argument (lines 279-288), he moves to trying to solve the problem using his preferred coordinate system, possibly using conservation of energy.

Wes's diversion into energy is not just fleeting whim here. When the problem is presented to the pair again in week 10, he brings up energy arguments again. Physicists find that energy conservation (as opposed to vector force summation) is an attractive solution method for many problems in physics. For a simple pendulum, energy is conserved as it swings to and fro. Solving for the speed of the bob as a function of position is therefore a trivial exercise. Using energy arguments, a natural coordinate system is Cartesian; gravitational potential energy, expressed as $m g h$, requires that one coordinate be vertical and measured with positive up. Wes is most likely thinking of these arguments when he defines his "starting height" in line 279.

Energy conservation arguments are alluring, but energy arguments cannot be employed to solve for the position as a function of time. In the second part of Wes's argument, he mentions that he would like to use energy, but then he gets stuck with that approach. Though he is not explicit, it may be that some of his difficulty stems from the inapplicability of energy arguments to this question of time. That is not the 
sole source of his difficulty with this problem, however, as he goes on to describe in the third part of his argument, included below.

Wes: (15:00) (Continued) And if you want position in $x, y$, then, it's gonna be...stupid to do. To try. It would be easier to do position as opposed to angle of displacement. (Hands marker to Derek, who begins writing)

290 TA: Does uh-

Wes: Rest point

TA: Does a position based on angle and how far you are away give you the same information as it's $x, y$ coordinates.

295 Wes: Well I can't think of any way to put it in terms of-you want what's this point. What are it's coordinates at this point?

TA: "Where is it?" "Where is it?"

Wes: But you'd have to do that in terms of

TA: Sure.

Wes: Sure.

TA: That's-

Wes: And, uh...so I would say I don't

know any functions that would give you

TA: Mmkay.

Wes: At the same time. (Inaudible) I mean, you could base it on $y$ and say ok and

TA: Okay.

Wes: (16:00) If you want a specific point, I would almost want to take the route of what's it's angle of displacement. But,

TA: Would displacement tell you exactly where it was? At any time?

Wes: Huh? Well, no, you'd have to figure that out. But,

In this third part of Wes's argument, Wes wonders if it would be smarter to measure position based on displacement from starting angle. The TA's question in lines 292-294 is a pointed question of equivalence and span. Wes does not address the equivalence argument. Instead, he explains that he "[does not] know any functions that would give you two parameters" and thus he cannot solve the two-dimensional problem using the "traditional $x-y$ " coordinate system. He seems to have activated span as a relevant measure of modeling but seems unable to connect ease and span together to point to his chosen coordinate system. The TA presses the point in line 307, asking if angular displacement is sufficient. Wes replies that he does not know and that he would "have to figure that out," further evidence that Wes's polar resource is not well connected to other resources. In particular, it seems connected to neither natural nor easy. Furthermore, it seems that while he has activated equivalence in order to consider using an alternate system, he is unable to fully switch to that system because it is neither natural nor easy.

When the equivalence question is put to Derek in lines $327-329$, he replies quickly that knowing $\theta$ as a function of
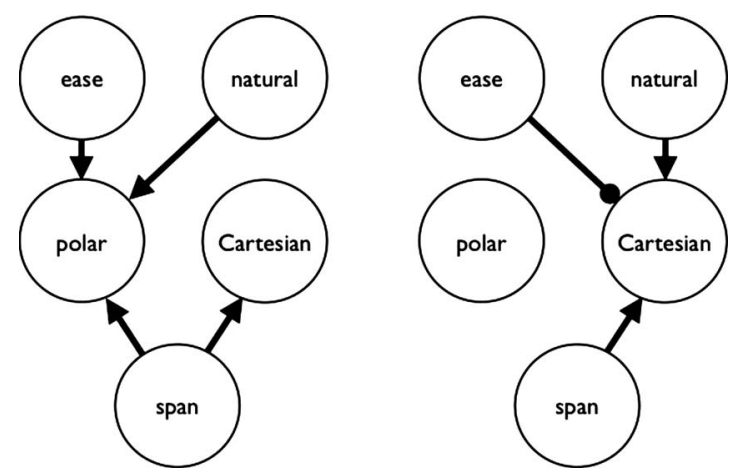

FIG. 5. Resource graph of Wes and Derek from the week 4 miniview. Note the explicit lack of connection between ease of calculation and Cartesian resources for Wes but not for Derek. Only natural, ease, Cartesian, polar, and span are shown.

time is sufficient. The quickness of his reply and the matterof-fact tone in which he delivers it are evidence that polar is more solid for Derek than it is for Wes. Derek's quickness of reply implies solidity here because it has a quickly activated connection to polar. However, when Wes quickly activates polar, without connections, it is recognized but plastic. This difference shows that cuing reliability alone is insufficient for determining plasticity; the other heuristics are necessary as well.

In contrast to Wes, Derek sees the span of polar and Cartesian coordinates as sufficient for this problem and sees polar coordinates as natural for this problem. We represent this description of resource use by Wes and Derek in Fig. 5. Note that one arrow is drawn to indicate the explicit lack of connection between Wes's ease and Cartesian resources.

\section{Origin}

Of particular interest in the discussion of Wes and Derek on coordinate system choice is their choice of origin. To examine the subtleties of their ideas, a brief interlude about the nature of the origin is necessary.

In all locational coordinate systems, locations are expressed in reference to the values of the coordinates. In a Cartesian system, the zeros of each coordinate (a statement of value) converge at one point (the origin) and that point can only be specified using all coordinates. Thus, origin is a combination of value and consistent in a Cartesian system. In a polar system, at the point where the radial coordinate is zero (analogous to the origin in Cartesian coordinates), the angular coordinate may take any value. In that respect, this point is unique in polar coordinates, and Cartesian coordinates do not have an analogous point that can be wholly specified using only one coordinate. Asking where the origin is-and meaning the place where all coordinates have a value of zero-when using polar coordinates is not a fair question.

Wes and Derek both activate origin resources, as evidenced by their discussion starting at line 183 when the TA asks them to apply their coordinate system to the sketch of the pendulum. Immediately following the $\theta$ direction and value discussion, they continue into a discussion of $\boldsymbol{r}$, speci- 
fying both the location for zero and direction.

Later, to further explain his span argument and equivalence confusion, Wes brings in a discussion of value. He restates his argument to Derek, who had been working privately the first time, reiterating that to get a "precise exact location in the $x$ and $y$," two equations are necessary, one for each coordinate. Each position is thus expressible in terms of the value of each coordinate. In polar, however, Wes has a problem with expressing locations:

Wes: But in polar, if you want it's exact location, in space, you have to have it in reference to something.

Derek: The origin.

345 Wes: So if you have it referenced to the origin, you know that this length is going to be constant, which is $r$ in this system, and so you could say it's anywhere there (in a circle of radius $L$ ).

Wes's difficulty in expressing the "exact location" of the bob may be related to the nature of the origin in polar coordinate systems. It seems here that he does not view polar coordinates as being a proper coordinate system, further evidence of polar's plasticity to him.

\section{Summary of week 4 discussion}

Derek and Wes are using different coordinate systems to describe the physical situation. For Derek, polar is appropriate because of the natural geometry and calculational ease. Wes thinks of Cartesian coordinates as "traditional" and natural and struggles with polar coordinates. We have described some of these two students' resources in terms of resource graphs and on a plasticity continuum.

It could be argued that Wes does not have a polar resource at all; the components of a polar system are too weakly connected to call polar a resource for him. Such an argument belies Wes's original work in determining the coordinate system. In line 184 , he refers to his coordinate system as "just polar," as if that is a sufficient response to the TA's request to tell her more about the system; earlier, he quickly names and labels his coordinates (lines 159-160), $\boldsymbol{r}$ and $\boldsymbol{\theta}$. By resource heuristic R2 (referable), polar is a resource. However, by plasticity heuristics P1 (ease of use), P3 (elaboration), and P5 (extended use) it is extremely plastic.

\section{B. Revisiting polar coordinates (week 10)}

In week 10, the task in Fig. 2 is posed to the students again. In the intervening weeks, students have studied damped and driven harmonic motion in class and have been assigned a homework problem on the equation of motion for the pendulum (derived using both Lagrangian and Newtonian methods). Their responses typify their approaches to the class: Wes says that he "tried to use radians but got stuck and gave up;" Derek says that to solve this problem, he would just "assume a solution."

When the TA asks them to define a coordinate system, Derek chooses a polar coordinate system for the same reasons he did in week 4 . Wes once again chooses a Cartesian

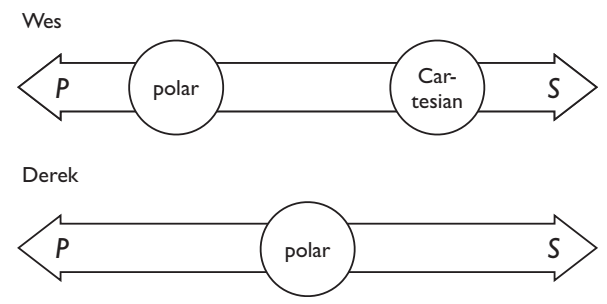

FIG. 6. Two plasticity charts for the week 4 miniview. Wes's graph is on top, Derek's below. Wes's polar resource is more plastic than both his Cartesian one and Derek's polar, but we do not have enough evidence to learn how solid Derek's polar is.

system. However, instead of choosing the standard system where positive is up and to the right as he did in week 4 , he tailors his system to the problem at hand, defining positive down and to the right. The downward direction is consistent with the weight vector, showing a better match of coordinates to physical situation. Because he tailors his coordinate system to the problem, rather than automatically choosing a generic system, his Cartesian resource has become more solid in this context.

At the TA's prompting, Wes continues to write Newton's second law for the system and starts to break the forces into components, defining $\theta$ as the angle between the horizontal and the position of the bob $\left(\theta_{1}\right.$ in Fig. 4). His choice of Cartesian coordinate system complicates the problem, and he gets stuck.

Derek uses Wes's confusion as evidence that a Cartesian system is inappropriate. As illustration, he writes Newton's second law and breaks it into $\mathbf{r}$ and $\theta$ components. Derek writes

$$
\begin{gathered}
\sum F_{x}=m \frac{d^{2} \theta_{x}}{d t^{2}}, \\
T \cos \theta=m \frac{d^{2} \theta_{x}}{d t^{2}} .
\end{gathered}
$$

After writing Eqs. (1) and (2), Derek reads them aloud. In reading them, he corrects himself: because $\theta$ is a coordinate in its own right, he does not need to use the subscript $x$. His equation, amended, reads

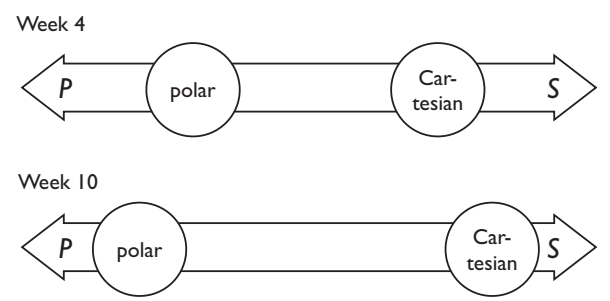

FIG. 7. A comparison of plasticity of polar and Cartesian for Wes in weeks 4 and 10. Cartesian has become more solid as his Cartesian choices become tailored to the problem at hand, while his polar resource seems more plastic. 


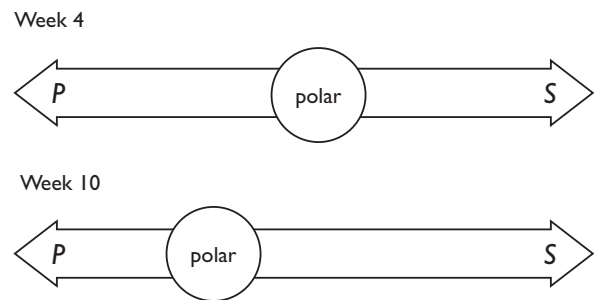

FIG. 8. A comparison of the plasticity of Derek's polar resource in weeks 4 and 10 . In week 4 , there was insufficient evidence to know how plastic polar is (only that it is more solid than Wes's). In week 10, Derek's derivation details indicate that it is plastic.

$$
T \cos \theta=m \frac{d^{2} \theta}{d t^{2}} .
$$

This equation differs from the standard physics equation because the angle defined as $\theta$ is the complement of the typically chosen angle. Furthermore, it is dimensionally inconsistent: the left-hand side has units of force and the righthand side has units of mass per time squared. These differences aside, Derek's equation has the right form for the differential equation.

An equation in place, the TA again asks the students to label their coordinates on their diagram. Derek first copies over Wes's definition of $\theta$ ( $\theta_{1}$ on Fig. 4$)$, then argues that by alternate interior angles, it is equal to $\theta_{2}$. The TA asks where $\theta$ is equal to zero, and Derek redefines $\theta$ to be $\theta_{3}$, the common and calculationally easy physicist response. When the TA asks the direction of $\theta$ at the instant shown, Derek argues that the bob is moving in a circular arc and that, at any point along the arc, $\theta$ is tangent to the arc. He draws $\hat{\boldsymbol{\theta}}_{\mathbf{4}}$. With all four $\theta$ definitions arrayed before him, Derek expresses doubt that he has a sensical answer, as shown in Fig. 6.

We interpret the evidence from the week 10 group interview to indicate even more strongly that Wes's polar resource is very plastic (Fig. 7). It is not well connected to other resources, in particular with calculational ease. Derek, whose polar resource seemed more solid than Wes's, shows evidence of problems with the ease of applying the natural coordinates for this problem, indicating that it is still plastic to him (Fig. 8). We present resource graphs of the resource use by Wes and Derek in Fig. 9.

\section{DISCUSSION}

In this paper, we introduce a theoretical structure by which we can understand the development of new resources and their connection to existing resources. We build from Resource Theory. One of the elements of Resource Theory is that resources can be nested, containing other resources. We represent some of this structure through resource graphs. We add ideas from Process/Object and RBC theory to help us develop observable tools for understanding the plasticity of resources as they develop over time. We identify four resource heuristics (R1-reusable, R2-referrable, R3-
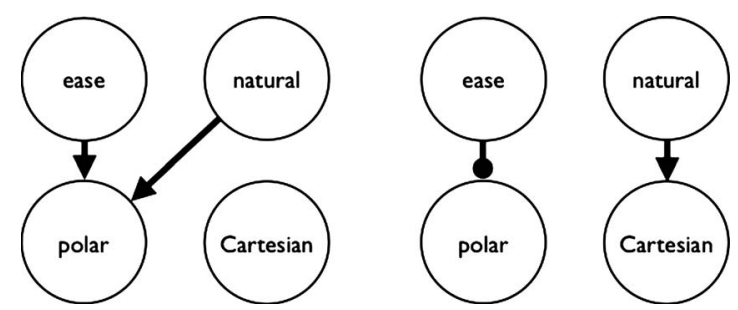

FIG. 9. Resource graphs of Wes and Derek for the week 10 miniview. Note the explicit lack of connection between ease and polar resources for Wes but not for Derek.

nameable, and R4-activatable) and five plasticity heuristics (P1-ease of use, P2-recency of construction, P3elaboration, P4 - justification, and P5-extended use) to aid in identifying resources and their plasticity. These heuristics arise as extensions of previous discussions of the development of primitives and resources.

To make a plausible argument for the validity of our construct of resource plasticity, we have applied our theoretical structure to help explain student reasoning about coordinate systems in a canonical physics problem that nevertheless presents difficulties to students. We have shown resource graphs of two students arguing about which coordinate system to use. We used these graphs to indicate the level of plasticity of two specific available coordinate systems (polar or Cartesian).

We find that students sometimes persist in using an inappropriate Cartesian system despite professed knowledge of polar coordinates, indicating that Cartesian coordinates are quite solid to these students. Furthermore, students must rederive (rather than recall) the details of the polar coordinate system, indicating that polar coordinates are quite plastic.

The plasticity continuum provides a measure for resources as they are being created. It extends Resource Theory to those situations when students struggle to learn ideas wholly unfamiliar to them and not describable in a primitive sense. Detailing the interactions between resources gives us better insight into the working of student minds and lets us build better models of our students and their learning. 49

\section{ACKNOWLEDGMENTS}

These results were presented in expanded form as part of the unpublished dissertation of ECS. ${ }^{50}$ We are grateful for the insight of and comments from John E Donovan II, Andrew Elby, and the members of the University of Maine Physics Education Research Laboratory. The interviews described in this paper were conducted by Katrina E. Black. This work was funded in part by NSF under Grants No. REC-0633951 and No. DUE-0442388.

\section{APPENDIX: SUPPLEMENTARY AUXILIARY MATERIAL}

See separate auxiliary material for complete transcript of the two interviews described in the main paper. 
${ }^{1}$ A. Sfard, On the dual nature of mathematical conceptions: Reflections on process and objects as different sides of the same coin, Educ. Stud. Math. 22, 1 (1991).

${ }^{2}$ D. Hammer, Student resources for learning introductory physics, Am. J. Phys. 68, S52 (2000).

${ }^{3}$ We see Resource Theory as being a theoretical umbrella under which many subtheories may shelter. Some of the subtheories underneath resources include p-prims (Ref. 10), symbolic forms (Ref. 11), and coordination classes (Ref. 23). We denote umbrellas as "Theories" and those underneath them as "theories" (note capitalization). Thus, Resource Theory and Process/Object theory are umbrellas which shelter plasticity and RBC theory (respectively).

${ }^{4}$ R. Hershkowitz, B. B. Schwarz, and T. Dreyfus, Abstraction in context: Epistemic actions, J. Res. Math. Educ. 32, 195 (2001).

${ }^{5} \mathrm{P}$. Tsamir and T. Dreyfus, Comparing infinite sets-a process of abstraction: The case of Ben, J. Math. Behav. 113, 1 (2002).

${ }^{6} \mathrm{~T}$. Dreyfus and P. Tsamir, Ben's consolidation of knowledge structures about infinite sets, J. Math. Behav. 23, 271 (2004).

${ }^{7} \mathrm{P}$. Tsamir and T. Dreyfus, How fragile is consolidated knowledge?, J. Math. Behav. 24, 15 (2005).

${ }^{8}$ M. S. Sabella and E. F. Redish, Knowledge organization and activation in physics problem solving, Am. J. Phys. 75, 1017 (2007).

${ }^{9}$ E. F. Redish, Proceedings of the International School of Physics Enrico Fermi, Course CLVI: Research on Physics Education, edited by E. F. Redish and M. Vicentini (IOS, Amsterdam, 2004), pp. 1-56.

${ }^{10}$ A. A. diSessa, Towards an epistemology of physics, Cogn. Instruct. 10, 105 (1993).

${ }^{11}$ B. L. Sherin, How students understand physics equations, Cogn. Instruct. 19, 479 (2001).

${ }^{12}$ Forms are general (mathematical) elements of a structure, which students apply to specific situations.

${ }^{13}$ D. Hammer and A. Elby, Tapping epistemological resources for learning physics, J. Learn. Sci. 12, 53 (2003).

${ }^{14}$ D. Hammer, E. F. Redish, A. Elby, and R. E. Scherr, in Transfer of Learning: Research and Perspectives, edited by J. Mestre (Information Age, Greenwich, CT, 2004).

${ }^{15}$ L. Lising and A. Elby, The impact of epistemology on learning: A case study from introductory physics, Am. J. Phys. 73, 372 (2005)

${ }^{16}$ E. C. Sayre, M. C. Wittmann, and J. R. Thompson, Physics Education Research Conference Proceedings 2003, edited by K. C. Cummings, S. Franklin, and J. Marx (Springer, New York/LLC, Secaucus, NJ, 2003).

${ }^{17}$ J. Tuminaro, Ph.D. thesis, University of Maryland, 2004.

${ }^{18}$ M. C. Wittmann, Using resource graphs to represent conceptual change, Phys. Rev. ST Phys. Educ. Res. 2, 020105 (2006).

${ }^{19}$ M. C. Wittmann and J. T. Morgan, Physics Education Research Conference Proceedings 2003, edited by S. Franklin, K. C. Cummings, and J. Marx (Springer, New York/LLC, Secaucus, NJ, 2004).

${ }^{20}$ D. Hammer and A. Elby, Fourth International Conference of the Learning Sciences, edited by B. Fishman and S. O'ConnerDivelbiss (Erlbaum, Mahwah, NJ, 2000), pp. 4-5.

${ }^{21}$ A. Elby and D. Hammer, On the substance of a sophisticated epistemology, Sci. Educ. 85, 554 (2001).

${ }^{22}$ K. E. Black and M. C. Wittmann, Physics Education Research Conference 2007 (AIP Conference Proceedings) No. 951 (AIP,
New York, 2007).

${ }^{23}$ A. A. diSessa and B. L. Sherin, What changes in conceptual change, Int. J. Sci. Educ. 20, 1155 (1998).

${ }^{24}$ J. Tuminaro and E. F. Redish, Elements of a cognitive model of physics problem solving: Epistemic games, Phys. Rev. ST Phys. Educ. Res. 3, 020101 (2007).

${ }^{25}$ R. S. Russ, Ph.D. thesis, University of Maryland, 2006.

${ }^{26}$ A. Elby, Helping physics students learn about learning, Am. J. Phys. 69, S54 (1999).

${ }^{27}$ M. C. Wittmann and J. T. Morgan, Intuitive quantum physics, http://perlnet.umaine.edu/iqp/

${ }^{28}$ B. S. Ambrose, Investigating student understanding in intermediate mechanics: Identifying the need for a tutorial approach to instruction, Am. J. Phys. 72, 453 (2004).

${ }^{29}$ M. C. Wittmann and B. S. Ambrose, Intermediate mechanics tutorials, http://perlnet.umaine.edu/imt/

${ }^{30}$ Though individual resources are held by individuals, their expression and use in a specific context may be socially negotiated. However, we wish to draw a distinction between resources (held individually) and their appropriate expression (which may be socially determined).

${ }^{31}$ This is, of course, a simplification. We do not discuss here an important third state-primed-for either neurons or resources.

${ }^{32}$ D. Tannen and C. Wallat, in The Discourse Reader, edited by A. Jaworski and N. Coupland (Routledge, New York, 1987), pp. 346-366.

${ }^{33}$ E. C. Sayre, M.S. thesis, University of Maine, 2005.

${ }^{34}$ However, if a resource activates in two contexts, those contexts may or may not be near each other. Consider the conservation of stuff resource. In quantum mechanics, the energy of a particle is conserved as it passes through a barrier, though students may think the energy decreases (Ref. 19). In a classic Piagetian experiment, water is poured from a short fat glass to a tall skinny one, but its volume remains the same, though children may think the volume increases (Ref. 51). In both cases, conservation of stuff activates appropriately, but the cases bear no other resemblance. Thus, conservation of stuff could be nearby many otherwise disparate resources. Yet, if those other resources are disparate, they should not be near each other. To solve the addressing difficulty, resources need to be in two "places" at the same time. Obviously, ordinary buildings cannot have multiple locations.

${ }^{35}$ For a overview, see McDermott and Redish, 1999 (Ref. 52).

${ }^{36}$ B. L. Sherin, Ph.D. dissertation, University of California, 1996.

${ }^{37}$ J. Minstrell, in Research in Physics Learning: Theoretical Issues and Empirical Studies, Proceedings of an International Workshop, Bremen, Germany, 1991, edited by R. Duit, F. Goldberg, and H. Niedderer (IPN, Kiel, 1992), pp. 110-128.

${ }^{38}$ S. Demastes, R. Good, and P. Peebles, Patterns of conceptual change in evolution, J. Res. Sci. Teach. 33, 407 (1996).

${ }^{39}$ Minsky (Refs. 53,54) uses a similar but distinct definition for frames. He is much closer to what we call a resource graph, in which ideas are recruited into the frame system, and we do not discuss it further here.

${ }^{40}$ For example, one might consider when certain framing activities are possible and how that depends on the readout strategies associated with a given resource graph. Perhaps only those resource graphs with appropriate readouts are available to the activity of framing. However, there is too little theoretical clarity on either end of the description for a connection to be made. Under what conditions is one unable to frame an activity in a 
certain way? What does it mean for a readout strategy to be unavailable in a given setting? Such questions must be answered before appropriate connections can be made between our discussion here and framing in a resources perspective.

${ }^{41}$ E. C. Sayre, M. C. Wittmann, and J. E. Donovan, in Physics Education Research Conference 2007 (AIP Conference Proceedings) No. 883, edited by L. McCullough, L. Hsu, and P. R. Heron (Springer, New York,/LLC, Secaucus, NJ, 2007), pp. 8588.

${ }^{42}$ R. E. Scherr, Modeling student thinking: An example from special relativity, Am. J. Phys. 75, 272 (2007).

${ }^{43}$ The purpose of these heuristics is not to make a list of possible resources but to understand the resources in use by a single student. However, in situations where many students make the same statement with only slight variations, one may posit that a resource common to all may be in use even if that resource might be quite plastic (Ref. 55).

${ }^{44}$ It is a somewhat philosophical question to wonder whether a resource exists which has never activated. Because resources are defined as activatable, if it could activate (and merely has not), then it might exist.

${ }^{45}$ R. Brown and J. Kulik, Flashbulb memories, Cognition 5, 73 (1977).
${ }^{46}$ B. S. Ambrose, Incorporating a tutorial approach in an advanced mechanics course for physics majors, Announcer 33, 124 (2003).

${ }^{47}$ G. R. Fowles and G. L. Cassiday, Analytical Mechanics, 7th ed. (Brooks-Cole, Belmont, MA, 2004).

${ }^{48}$ The tension is not constant, in conflict with Derek's claim in line 146.

${ }^{49}$ Note that while these coordinates are not necessarily positions, the language used to describe their system is locationlike.

${ }^{50}$ E. Sayre, Ph.D. thesis, University of Maine, 2007.

${ }^{51} \mathrm{~J}$. Piaget and B. Inhelder, The Child's Conception of Space (Norton, New York, 1967).

${ }^{52}$ L. C. McDermott and E. F. Redish, Resource letter PER-1: Physics education research, Am. J. Phys. 67, 755 (1999).

${ }^{53}$ M. Minsky, in The Psychology of Computer Vision, edited by P. Winston (McGraw-Hill, New York, 1975).

${ }^{54}$ M. Minsky, The Society of Mind (Simon and Shuster, New York, 1985).

${ }^{55}$ D. B. Harlow and V. K. Otero, in Physics Education Research Conference Proceedings 2005 (AIP Conference Proceedings) No. 818, edited by P. L. Heron, L. McCullough, and J. Marx (Springer, New York/LLC, Secaucus, NJ, 2006). 